\title{
Gastric rupture as a rare complication in diagnostic upper gastrointestinal endoscopy
}

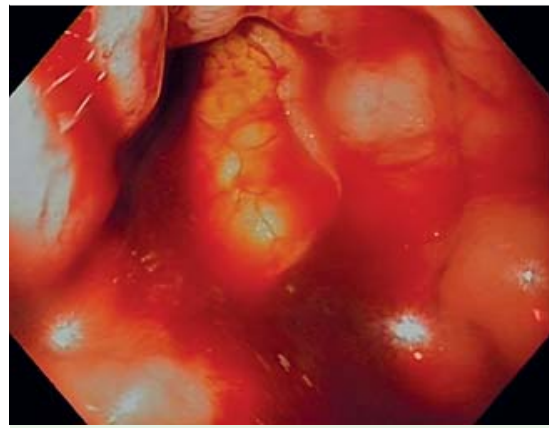

Fig. 1 Endoscopic image in a 76-year-old woman undergoing diagnostic esophagogastroduodenoscopy showing a large perforation on the lesser curvature of the gastric body with profuse bleeding and visible intra-abdominal fat.

A 76-year-old woman was referred for diagnostic esophagogastroduodenoscopy (EGD) because of melena and iron deficiency anemia without other symptoms of gastrointestinal disease. Although the patient was a smoker, she had no major signs of chronic obstructive lung disease with an oxygen saturation of $90 \%$. EGD with conscious sedation using midazolam was performed by a physician in training and supervised by an expert gastroenterologist. Esophageal intubation went smoothly and the stomach was carefully insufflated with room air to show normal mucosa. Only modest signs of inflammation and erosions were detected in the duodenum. As the endoscope was being withdrawn, biopsies were taken in the antrum for Helicobacter pylori testing. At this point the patient suddenly started to cough and choke, upon which vigorous bleeding and mesenteric fat were observed in the proximal part of the lesser curvature of the gastric body, indicating that a perforation had occurred ( Fig. 1).

The examination was immediately terminated and the patient underwent emergency laparotomy, which revealed an 8-cm long, full-thickness perforation along the lesser curvature of the stomach $(\bullet$ Fig.2). The gastric perforation was sutured and the patient recovered uneventfully.

Diagnostic EGD is a very safe procedure with the reported incidence of perforation being below $0.05 \%$ [1]. Gastric perforations due to malignancy and therapeutic endoscopy may occur anywhere in the stomach [2]. In contrast, most cases of gastric rupture due to increased intra-abdominal pressure (cardiopulmonary resuscitation, Heimlich maneuver and blunt abdominal trauma), as well as over distension by food or hair, and barotrauma occur in the proximal part of lesser gastric curvature [3-5].

In the present case, it is assumed that the choking and coughing, possibly related to her smoking history and aspiration of saliva, together with insufflation-induced gastric distension led to increased intraabdominal pressure, thereby causing the gastric rupture. Indeed, vomiting and forceful coughing have been previously reported to provoke gastric rupture [4]. This case also indicates the need for careful attention to be paid to the airway at all

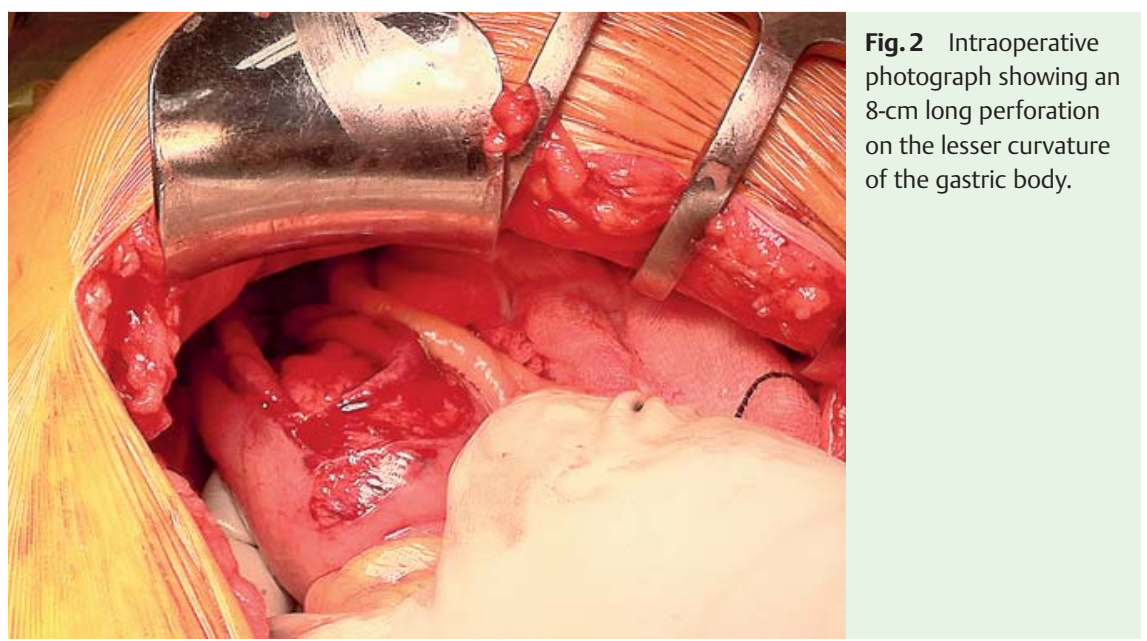

times during upper gastrointestinal endoscopy, in order to prevent choking and forceful vomiting.

Although gastric rupture is an exceptionally rare complication in diagnostic EGD, patients should be informed of the risk. Endoscopists should be aware of this severe and potentially life-threatening complication in all upper gastrointestinal endoscopic procedures.

Endoscopy_UCTN_Code_CPL_1AH_2AB

Competing interests: None

\section{Gabriele Wurm Johansson', Artur Nemeth ${ }^{1}$, Jörgen Nielsen ${ }^{1}$, Tobias Axmarker' ${ }^{2}$, Stefan Santen ${ }^{2}$, Henrik Thorlacius ${ }^{2}$, Ervin Toth ${ }^{1}$}

1 Endoscopy Unit, Department of Gastroenterology, Skåne University Hospital, Malmö, Sweden

2 Department of Surgery, Skåne University Hospital, Malmö, Sweden

\section{References}

1 Hart R, Classen M. Complications of diagnostic gastrointestinal endoscopy. Endoscopy 1990; 22: 229-233

2 Matsunaga $Y$, Goto A, Kaneko $H$ et al. Gastric rupture before puncture of the stomach in percutaneous gastrostomy. Endoscopy 2011; 43 (Suppl. 02): E57

3 Tung PH, Law S, Chu KM et al. Gastric rupture after Heimlich maneuver and cardiopulmonary resuscitation. Hepatogastroenterology 2001; 48: 109-111

4 Nakao A, Isozaki $\mathrm{H}$, Iwagaki $\mathrm{H}$ et al. Gastric perforation caused by a bulimic attack in an anorexia nervosa patient: report of a case. Surg Today 2000; 30: 435-437

5 Bharathan R, Datta S, Webb P et al. Trichobezoar and postpartum gastric perforation: a case report and brief review of literature. J Obstet Gynaecol 2008; 28: 438-440

Bibliography

DOI http://dx.doi.org/

10.1055/s-0033-1344873

Endoscopy 2013; 45: E391

(c) Georg Thieme Verlag KG

Stuttgart - New York

ISSN 0013-726X

Corresponding author

Gabriele Wurm Johansson, MD

Skåne University Hospital - Department

of Gastroenterology

Inga Marie Nilssons gata 49

Malmö 20502

Sweden

Fax: +4640338699

Gabriele.WurmJohansson@skane.se 